Pacific Journal of Mathematics

UNIMODULAR CONTRACTIONS IN HILBERT SPACE 


\title{
UNIMODULAR CONTRACTIONS IN HILBERT SPACE
}

\author{
Bernard Russo
}

Let $T$ be a unitary operator on a Hilbert space $H$. Then in particular,

(i) $T$ is a contraction, i.e. $\|T\| \leqq 1$; and

(ii) The spectrum of $T$ is a subset of the unit circle, i.e. $\operatorname{Sp}(T) \subset C$, where $C$ denotes the set of complex numbers of absolute value one.

Call an arbitrary operator $T$ a unimodular contraction if it satisfies conditions (i) and (ii) above. Then several questions immediately come to mind. Do there exist nonunitary unimodular contractions? If so, what is the nature of their spectra, e.g. what subsets of the unit circle arise as spectra of nonunitary unimodular contractions; when does the spectrum contain point, residual, or continuous spectrum? Under what conditions is a unimodular contraction unitary? What is the nature of operator algebras containing nonunitary unimodular contractions?

In this paper examples are given of nonunitary unimodular contractions. It is shown (Theorem 2) that such exist with arbitrarily prescribed spectrum, which however can contain no residual spectrum. It is also shown (Theorem 1 ) that nonunitary unimodular contractions exist only in infinite von Neumann algebras. This result is applied to a mapping problem of operator algebras.

1. Preliminary discussion. We first dispose of eigenvalues.

Lemma 1.1. Let $T$ be a unimodular contraction. Then eigenvectors of $T$ corresponding to distinct eigenvalues are orthogonal.

Proof. If $T x=r x, T y=t y$, where $x$ and $y$ are unit vectors and $r$ and $t$ are distinct complex numbers of absolute value one, then the expansion of $\|T(a x+b y)\| \leqq\|a x+b y\|$ yields $\operatorname{Re} r \bar{t} a \bar{b}(x, y) \leqq \operatorname{Re} a \bar{b}(x, y)$ for all complex numbers $a$ and $b$. Since $r \neq t$, this can happen only if $(x, y)=0$.

Proposition 1.2. If $T$ is a unimodular contraction on a Hilbert space $H$, whose eigenvectors span $H$, then $T$ is unitary.

Proof. The hypothesis and the preceding lemma imply that the eigenspaces of $T$ are orthogonal and that $H$ is their direct sum. It follows that $T$ is an isometry of $H$ onto $H$.

By reduction of a matrix to triangular form it is easily seen that a unimodular contraction on a finite dimensional Hilbert space is unitary. 
In the general infinite dimensional case it follows from the proof of Proposition 1.2 that by dropping to the orthogonal complement of the eigenspaces we may assume that the spectrum of a unimodular contraction is continuous. Indeed, since the spectrum is all boundary, it follows that $\mathrm{Sp}(T)$ coincides with the approximate point spectrum of $T$. Since by the above remarks, the point spectrum may be assumed empty, it follows that the spectrum of $T$ is all continuous spectrum. By contrast, we mention an example of Jamison [6]. He exhibits a nonnormal operator $T$ on $L^{2}(0,1)$ whose spectrum is the unit circle $C$ and consists of point spectrum only.

In general, except for Corollary 1, the author has been unable to find sufficient conditions for the normality of a unimodular contraction. In view of the result below (Theorem 2) and the preceding paragraph, the size or type of the spectrum is not the place to look.

\section{Examples and main results.}

EXAMPLE 1. Let $H$ be the set of sequences $a=\left\{a_{n}\right\}_{-\infty}^{\infty}$ of complex numbers such that $\Sigma\left|a_{n}\right|^{2}$ is finite, with the usual inner product $(a, b)=\Sigma a_{n} \bar{b}_{n}$. Let $\left\{x^{(n)}\right\}_{-\infty}^{\infty} \subseteq H$ be the canonical orthonormal basis for $H$, i.e. $x^{(n)}$ is the "characteristic function of $n$ ". Let $0<t<1$, and let $G$ be the operator defined by

$$
G x^{(n)}= \begin{cases}t x^{(0)} & \text { if } n=0, \\ x^{(n)} & \text { if } n \neq 0 .\end{cases}
$$

Let $U$ denote the bilateral shift operator, $U x^{(n)}=x^{(n+1)}$, for all $n$. Then $T=U G$ is a contraction which is not unitary. We assert that $T$ is a unimodular contraction. Indeed, a computation yields $\left\|T^{-k}\right\|=t^{-1}$ for $k=1,2, \cdots$. Thus the spectral radius of $T^{-1}$ is 1 . It follows that $\operatorname{Sp}(T)$ is a subset of the unit circle. Further investigation shows that $\operatorname{Sp}(T)$ is the entire unit circle.

The example $T$ just given cannot lie in any finite von Neumann algebra. The reason is that $T$ is completely nonunitary, i.e. for each nonzero vector $x$, there is a positive integer $n$ such that either $\left\|T^{n} x\right\| \neq\|x\|$, or $\left\|T^{* n} x\right\| \neq\|x\|$; but it is not true that the sequence $T, T^{2}, T^{3}, \cdots$ converges to 0 in the strong operator topology [3: p. 32]. On the other hand, a theorem of Foias and Kovács [4: Th. 1] states that a von Neumann algebra $M$ is finite if, and only if, $T^{n}$ converges strongly to 0 for each completely nonunitary contraction $T$ in $M$.

In what follows certain concepts from the theory of von Neumann algebras are used. A standard reference is [3]. For the convenience of the reader these concepts are summarized here.

A von Neumann algebra is a self-adjoint algebra of bounded linear operators on a Hilbert space which contains the identity operator and 
is closed in the weak operator topology [3: p. 33]. A von Neumann algebra $M$ is finite if for each $T$ in $M, T^{*} T=I$ (the identity) implies $T T^{*}=I$. A trace on a von Neumann algebra $M$ is a function $\varphi$ defined on the positive operators in $M$ with extended nonnegative real values which satisfies the conditions (i) $\varphi(S+T)=\varphi(S)+\varphi(T)$ (ii) $\varphi(r T)=$ $r \varphi(T)$ if $r \geqq 0$, (iii) $\varphi\left(U T U^{*}\right)=\varphi(T)$ for $U$ unitary in $M$. A trace $\varphi$ is faithful if $T \geqq 0$ and $\varphi(T)=0$ imply $T=0$, normal if $\varphi\left(\operatorname{LUB}_{r} T_{\gamma}\right)=$ $\mathrm{LUB}_{\gamma} \varphi\left(T_{\gamma}\right)$ for each norm bounded increasing family $\left\{T_{\gamma}\right\}$ of positive operators in $M$, and finite if the range of $\phi$ does not contain infinity. If $M$ is a von Neumann algebra with a finite trace $\varphi$ such that $\varphi(I)=1$, the determinant function $\Delta$ is defined on the set of regular elements of $M$ by the formula $\Delta(T)=\exp \varphi(\log |T|)$, where $|T|=\left(T^{*} T\right)^{1 / 2}$ [3: p. 105]. The properties of $\Delta$ that we shall use are (i) $\Lambda(S T)=\Delta(S) \Delta(T)$, (ii) $\Delta(T) \leqq \sup \{|z|: z \in \operatorname{Sp}(T)\}$. We say that projections $P$ and $Q$ in a von Neumann algebra $M$ are equivalent if there is an element $X$ in $M$ such that $X^{*} X=P$ and $X X^{*}=Q$. A projection $P$ in $M$ is finite if there is no projection $Q \leqq P$ which is equivalent to $P$ (except $P$ ), and infinite otherwise. A von Neumann algebra is purely infinite if each nonzero projection belonging to its center is infinite.

Lemma 2.1. Let $M$ be a von Neumann algebra with a faithful finite normal trace $\varphi$ such that $\varphi(I)=1$. Then each contraction $T$ in $M$ of determinant 1 is unitary.

Proof. Since $\|T\| \leqq 1,0 \leqq|T| \leqq I$ so that $\log |T| \leqq 0$. But $1=\exp \varphi(\log |T|)$ entails $0=\varphi(\log |T|) . \quad$ By faithfulness, $\log |T|=0$, $|T|=I, T^{*} T=I$. By finiteness of $M, T$ is unitary.

Lemma 2.2. Let $M$ be a von Neumann algebra with a faithful finite normal trace $\phi$ such that $\varphi(I)=1$. Then each unimodular contraction $T$ in $M$ is unitary.

Proof. It suffices to show that each unimodular contraction $T$ in $M$ has determinant 1. Since $\operatorname{Sp}\left(T^{-1}\right)=\operatorname{Sp}(T)^{-1}, T$ and $T^{-1}$ both have spectral radius 1. Thus $1=\Delta(I)=\Delta\left(T T^{-1}\right)=\Delta(T) \Delta\left(T^{-1}\right) \leqq 1$, so that \lrcorner$(T)=1$.

Lemma 2.3. Each purely infinite von Neumann algebra $M$ contains a nonunitary unimodular contraction.

Proof. By a result of Kaplansky [7: Lemma 4.4] there are projections $\left\{P_{n}\right\}_{-\infty}^{\infty}$ in $M$ which are mutually orthogonal and mutually equivalent, and $\Sigma P_{n}=I$. Let $H_{n}$ be the range of $P_{n}$. There is a unitary operator $U$ in $M$ such that $U H_{n}=H_{n+1}$ for all $n$. Let $0<t<1$. 
and set $G=t P_{0}+\left(I-P_{0}\right)$. The operator $T=U G$ is a nonunitary unimodular contraction in $M$ (cf. Example 1).

THEOREM 1. In order that a von Neumann algebra $M$ be finite, it is necessary and sufficient that each unimodular contraction in $M$ be unitary.

Proof. Suppose first that $M$ is finite. It is known [3: p. 98] that $M$ is a central direct sum of countably decomposable finite von Neumann algebras (each family of orthogonal projections is countable). It is also known [3: p. 98] that each countably decomposable finite von Neumann algebra has a faithful finite normal trace. Thus there are mutually orthogonal central projections $C_{\alpha}$ in $M$ such that $I=\Sigma_{\alpha} C_{\alpha}$ and $M=\Sigma_{n} M_{\alpha}$, where $M_{\alpha}=C_{\alpha} M$ has a faithful finite normal trace. If $T$ is a unimodular contraction in $M$, then $C_{\alpha} T$ is a unimodular contraction in $M_{\alpha}$. By Lemma 2.2, $C_{\alpha} T$ is unitary. Hence $T=\Sigma_{\alpha} C_{\alpha} T$ is unitary.

Conversely suppose that $M$ is not finite. Let $C$ be a maximal finite central projection in $M$ [3: p.97]. Since $M$ is not finite, $C \neq I$. Thus $M=C M \oplus(I-C) M$, where $C M$ is finite, and $(I-C) M$ is purely infinite. By Lemma 2.3, $(I-C) M$ contains a unimodular contraction $S$ which is not unitary. Then $C+S$ is a unimodular contraction in $M$ which is not unitary.

CoRollary 1. A unimodular contraction of finite type is unitary. (An operator is said to be of finite type if the von Neumann algebra it generates is finite).

As is known (see [10]), each contraction $T$ on Hilbert space $H$ has a unitary dilation $U$ defined on a Hilbert space $K$ containing $H$ as a closed subspace, i.e. $T x=P U x$ for $x$ in $H$, where $P$ is the projection of $K$ onto $H$. The unitary operator $U$ and the Hilbert space $K$ may be chosen to satisfy one or both of the following conditions:

(i) $T^{n} x=P U^{n} x, \quad T^{* n} x=P U^{-n} x$, for each $x$ in $H$ and each $n=0,1,2, \cdots$,

(ii) $K$ is the subspace generated by $U^{n} x$, with $x$ in $H$ and $n=0, \pm 1, \pm 2, \cdots$. The operator $U$ is correspondingly referred to as a strong unitary dilation of $T$, and a minimal unitary dilation of $T$, respectively. If $U$ is a strong unitary dilation of $T$, the spectral measure of $U$ gives rise to what is called a strong operator measure $F$ of $T$, namely for each Borel subset of $C$ (the unit circle), say $\sigma, F(\sigma) x=P E(\sigma) x, x$ in $H$, where $E$ is the spectral measure of $U$. By the support of $F$ (resp. $E$ ), notation $\Lambda(F)$ (resp. $\Lambda(E)$ ), is meant the complement of the union of all open sets of $F$-measure zero (resp. $E$-measure zero). Since $E(\sigma)=0$ implies $F(\sigma)=0$, we have $\Lambda(F) \subseteq \Lambda(E)$. 
M. Schreiber [10: Th. 2] has shown that $T$ is unitary if $\Lambda(F) \neq C$. The spectral measure $E$ of $U$ is supported on $\operatorname{Sp}(U)$. Hence we may state:

Proposition 2.4. Let $T$ be a contraction with a strong (not necessarily minimal) unitary dilation $U$. If $\operatorname{Sp}(U) \neq C$, then $T$ is unitary.

This result is also found in [12: Corollaire 2.2].

Examples of minimal unitary dilations of contractions which are not strong unitary dilations are provided by the Laurent and Toeplitz operators $L_{f}$ and $T_{f}$, where $f$ is a bounded measurable function on the unit circle, of absolute value 1 a.e., and for which neither $f$ nor $\bar{f}$, the complex conjugate of $f$, belongs to $H^{\infty}[1]$.

ExAmple 2. Let $T_{f}$ be the Toeplitz operator on $H^{2}$ of the unit circle defined by the function

$$
f\left(e^{i t}\right)= \begin{cases}e^{2 i t} & 0 \leqq t \leqq \pi \\ e^{-2 i t} & \pi \leqq t<2 \pi\end{cases}
$$

Then $T_{f}$ is a contraction such that $\operatorname{Sp}\left(T_{f}\right)=C$ (see [11: p. 286]). Observe that neither $f$ nor $\bar{f}$ belongs to $H^{\circ}$. It is known [1: p. 98] that the only Toeplitz operators which are unitary operators are the scalars of absolute value one.

Proposition 2.5. Let $T_{f}$ be a Toeplitz operator which is a unimodular contraction. Suppose that either $f$ or $\bar{f}$ belongs to $H^{\infty}$. Then either $T_{f}$ is a unitary operator or $\operatorname{Sp}\left(T_{f}\right)=C$.

Proof. By the theorem of Hartman and Wintner [5], Sp $\left(L_{f}\right)$ is a subset of $\operatorname{Sp}\left(T_{f}\right)$. Hence $L_{f}$ is a unitary dilation of $T_{f}$. Since either $f$ or $\bar{f}$ belongs to $H^{\infty}$, it follows from results in [1] that $L_{f}$ is a strong unitary dilation of $T_{f}$. By Proposition 2.4, if $T_{f}$ is not unitary, then $\operatorname{Sp}\left(L_{f}\right)=C$.

Example 3. Let $T$ be the operator on $L^{2}(0, \infty)$ defined by the formula $(T f)(x)=f(x)-x^{-1} \int_{0}^{x} f(t) d t$. Then $T$ is not unitary but it is shown in [2: p. 136-7] that $T$ is a contraction with $\operatorname{Sp}(T)=C$.

In each of the preceding three examples the spectrum of the nonunitary unimodular contraction $T$ was the entire unit circle $C$. That this was an accident is indicated by the following result. 
THEOREM 2. Let $K$ be a compact nonempty subset of the unit circle. Then there exists a nonunitary unimodular contraction $A$ such that $\operatorname{Sp}(A)=K$.

Proof. We begin with the special case in which $K$ consists of a single point, say $z_{0}$. On $L^{2}(0,1)$ let $V$ be the Volterra operator, $(V f)(x)=\int_{0}^{x} f(t) d t$. Then, as is known, $\operatorname{Sp}(V)=\{0\}$, and $V+V^{*}=P$ where $P f$ is the constant function $\int_{0}^{1} f(t) d t$. If $T$ is the Cayley transform of $i V$, i.e. $T=(V-I)(V+I)^{-1}$, then $\operatorname{Sp}(T)=\{-1\}$ and $I-T^{*} T=D^{*} P D$ where $D=2^{1 / 2}(V+I)^{-1}$. Since $P$ is positive, so is $I-T^{*} T$ so that $T$ is a contraction. The required operator is $-z_{0} T$. To get the general case, let $U$ be any unitary operator with spectrum equal to $K$. Fixing any point $z_{0}$ in $K$ and $T$ as above $A=U \oplus\left(-z_{0} T\right)$ is a nonunitary unimodular contraction with spectrum equal to $K$.

Retain the notation of the preceding paragraph. Since

$$
\operatorname{Im}(T)=-2(I+V)^{*-1} \operatorname{Im}(V)(I+V)^{-1}
$$

and $\operatorname{Im}(V)$ is compact it follows that $\operatorname{Im}(T)$ is compact, where $\operatorname{Im}(A)=A-A^{*} / 2 i$ for any bounded operator $A$. Hence the operators $T$ and $A$ of Theorem 2 are of type I, i.e. generate a von Neumann algebra of type I [13: Th. 2]. Moreover by Theorem $1 A$ is an operator of type $I_{\infty}$. By Theorem 1 every infinite von Neumann algebra contains nonunitary unimodular contractions.

Problem. Do there exists unimodular contractions of type $\mathrm{II}_{\infty}$ or III?

3. Linear mappings of operator algebras. In [8: Th. 2] it was shown that an identity conserving linear mapping of a von Neumann algebra $M$ into a von Neumann algebra $N$ which maps the semigroup of regular contractions in $M$ into the semigroup of regular contractions in $N$ is a $C^{*}$-homomorphism. We can now prove a similar result allowing $M$ to be merely a $C^{*}$-algebra provided that $N$ lies in some finite von Neumann algebra. If $\dot{\phi}$ is such a mapping, then the results of [8] show that $\phi$ maps the unitary operators in $M$ into unimodular contractions in $N$. By Theorem $1 \phi$ preserves unitary operators so is a $C^{*}$-homomorphism [9: Corollary 2].

\section{REFERENCES}

1. A. Brown and P. R. Halmos, Algebraic properties of Toeplitz operators, Jour. Reine Angw. Math 213 (1963), 89-102.

2. A. Brown, P. R. Halmos and A. L. Shields, Cesaro operators, Acta Sci. Math 26 (1965), 125-138. 
3. J. Dixmier, Les algebres d'operateurs dans l'espace Hilbertien, Gauthier-Villars, Paris, 1957.

4. C. Foias and I. Kovacs, Une caractérisation nouvelle des algèbres de von Neumann finies, Acta Sci. Math. 23 (1962), 274-278.

5. P. Hartman and A. Wintner, On the spectra of Toeplitz matrices, Amer. J. Math 72 (1950), 359-366.

6. B. Jamison, Eigenvalues of modulus 1, Proc. Amer. Math Soc. 16 (1965), 375-377.

7. I. Kaplansky, Projections in Banach algebras, Ann. of Math. 53 (1951), 235-249.

8. B. Russo, Linear mappings of operator algebras, Proc. Amer. Math Soc. 17 (1966), 1019-1022.

9. B. Russo and H. A. Dye, $A$ note on unitary operators in $C^{*}$-algebras, Duke Math. J. 33 (1966), 413-416.

10. M. Schreiber, A functional calculus for general operators in Hilbert space, Trans. Amer. Math. Soc. 87 (1958), 108-118.

11. - Numerical range and spectral sets, Michigan Math. J. 10 (1963), 283-288. 12. B. Sz.-Nagy and C. Foias, Sur les contractions de l'espace de Hilbert, III, Acta Sci. Math. 19 (1958), 26-45.

13. N. Suzuki, Algebraic aspects of non-self adjoint operators, Proc. Japan Acad. 41 (1965), 706-710.

Received November 14, 1966. This research was supported by a National Science Foundation grant. This paper contains portions of the author's doctoral dissertation submitted at the University of California, Los Angeles, January, 1965, under the supervision of Professor H. A. Dye.

UNIVERSITY OF CALIFORNIA

IRVINE, CALIFORNIA 



\section{PACIFIC JOURNAL OF MATHEMATICS}

\section{EDITORS}

\author{
H. ROYDEN \\ Stanford University \\ Stanford, California \\ R. R. Phelps \\ University of Washington \\ Seattle, Washington 98105
}

J. DUGUNDJI

Department of Mathematics

University of Southern California

Los Angeles, California 90007

RICHARD ARENS

University of California

Los Angeles, California 90024

\section{ASSOCIATE EDITORS}
E. F. BECKENBACH
B. H. NeumanN
F. WOLF
K. YosidA

\section{SUPPORTING INSTITUTIONS}

UNIVERSITY OF BRITISH COLUMBIA

CALIFORNIA INSTITUTE OF TECHNOLOGY

UNIVERSITY OF CALIFORNIA

MONTANA STATE UNIVERSITY

UNIVERSITY OF NEVADA

NEW MEXICO STATE UNIVERSITY

OREGON STATE UNIVERSITY

UNIVERSITY OF OREGON

OSAKA UNIVERSITY

UNIVERSITY OF SOUTHERN CALIFORNIA
STANFORD UNIVERSITY

UNIVERSITY OF TOKYO

UNIVERSITY OF UTAH

WASHINGTON STATE UNIVERSITY

UNIVERSITY OF WASHINGTON

AMERICAN MATHEMATICAL SOCIETY CHEVRON RESEARCH CORPORATION TRW SYSTEMS

NAVAL WEAPONS CENTER

Printed in Japan by International Academic Printing Co., Ltd., Tokyo, Japan 


\section{Pacific Journal of Mathematics}

\section{Vol. 26, No. $1 \quad$ November, 1968}

Efraim Pacillas Armendariz, Closure properties in radical theory......... 1

Friedrich-Wilhelm Bauer, Postnikov-decompositions of functors .......... 9

Thomas $\mathrm{Ru}-$ Wen Chow, The equivalence of group invariant positive definite

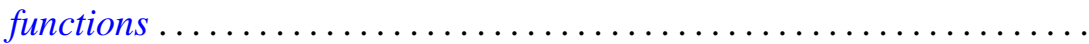

Thomas Allan Cootz, A maximum principle and geometric properties of

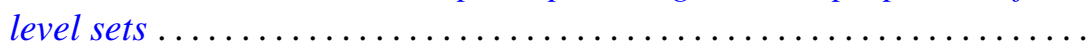

Rodolfo DeSapio, Almost diffeomorphisms of manifolds ............ 47

R. L. Duncan, Some continuity properties of the Schnirelmann density......

Ralph Jasper Faudree, Jr., Automorphism groups of finite subgroups of

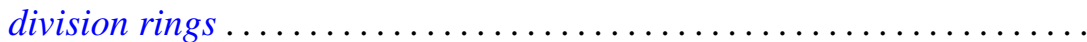

Thomas Alastair Gillespie, An invariant subspace theorem of $J$.

Feldman.........................................

George Isaac Glauberman and John Griggs Thompson, Weakly closed direct factors of Sylow subgroups .............................

Hiroshi Haruki, On inequalities generalizing a Pythagorean functional equation and Jensen's functional equation .....................

David Wilson Henderson, D-dimension. I. A new transfinite dimension.....

David Wilson Henderson, D-dimension. II. Separable spaces and

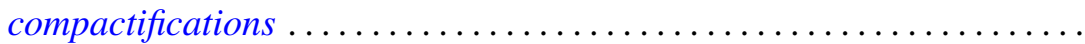

Julien O. Hennefeld, A note on the Arens products ............... 115

Richard Vincent Kadison, Strong continuity of operator functions ...

J. G. Kalbfleisch and Ralph Gordon Stanton, Maximal and minimal coverings of $(k-1)$-tuples by $k$-tuples.

Franklin Lowenthal, On generating subgroups of the Moebius group by pairs of infinitesimal transformations...

Michael Barry Marcus, Gaussian processes with stationary increments possessing discontinuous sample paths . .

Zalman Rubinstein, On a problem of Ilyeff ...

Bernard Russo, Unimodular contractions in Hilbert space. ...

David Lee Skoug, Generalized Ilstow and Feynman integrals...

William Charles Waterhouse, Dual groups of vector spaces . 